\title{
Elevation easier than plan for sighted and early-blind adults in a perspective-taking task
}

\author{
Hsin-Yi Chao • John M. Kennedy • Marta Wnuczko
}

Published online: 8 May 2013

(C) Psychonomic Society, Inc. 2013

\begin{abstract}
Plans show shapes of objects from above, and represent both their left-right order and their order in the $z$-dimension (the distance of the objects). Elevations show only the vertical shapes of objects arranged from left to right. Plans, having more spatial information, may be more difficult for participants to construct. Results from a study with sighted, sighted-blindfolded, and early-blind participants on Piaget's perspective-taking three-mountain task support this hypothesis. The plan task was judged more difficult than the elevation task even when participants performed with the same level of accuracy on both tasks. In visual and tactile tasks, amount of spatial-order information may determine difficulty, rather than plan versus elevation per se.
\end{abstract}

Keywords Perspective taking - Elevation and plan . Difficulty judgment $\cdot$ Early blindness $\cdot$ Touch

Here, we test hypotheses about perspective taking in earlyblind and sighted adults representing objects in a tabletop display. Making a plan of the layout required showing leftright and front-back locations, as in a 2-D map. Elevations show only the left-right order of the objects from an observer's point of view, and so plans may be more challenging than elevations. The hypothesis applies to sighted observers gathering information visually and also, we argue, to early-

\section{H.-Y. Chao}

National Taiwan University of Science and Technology,

Taipei, Taiwan

J. M. Kennedy $(\bowtie) \cdot$ M. Wnuczko

University of Toronto at Scarborough, 1265 Military Trail,

Toronto, ON M1C1A4, Canada

e-mail: kennedy@utsc.utoronto.ca blind and blindfolded participants reaching out and touching target objects.

Soska and Johnson (2008) noted that Piaget (1954) pioneered studies of perspective taking, often using the three-mountain task, which uses four different perspectives, at right angles to one another, on objects on a tabletop (Piaget \& Inhelder, 1956). The objects can be a cube, a sphere, and a cone, shown in Fig. 1a by a square, a circle, and a triangle (the cone from the side) or a circle with a dot in the center (the cone from above). Since occlusion is not an issue, from the participant's vantage point, in an elevation they are drawn in 2-D as a row, the circle on the left, the triangle in the middle, and the square on the right. A participant reports the objects from his or her own position and three others that are also without occlusion issues - ones to the left, right, and opposite. Another report can be a plan (Fig. 1b), a "view from above." The lower horizontal line of the plan indicates the observer's location. From that vantage point the cone, shown by the circle with a dot in the center, is the farthest object, shown by it being highest in the figure. The objects can be arranged so that each perspective is unique; for example, from opposite, the elevation would be shown by a row with the square on the left, the triangle in the middle, and the circle on the right.

The participant groups in the present study are split on the basis of whether visual information is available. In so doing, there are two different questions that emerge about accessing information about space: (1) what role vision plays and (2) what information haptics alone can play. Like vision and audition, haptics accesses spatial information for distal objects and egocentric location in the spatial array in many ways (Wolbers \& Hegarty, 2010), using pressure-driven cutaneous information (Vandoorn, Hohwy, \& Symmons, 2012), exploratory procedures with joints and muscles offering direct 
contact with objects (Klatzky \& Lederman, 2003), moments of inertia accessed by pendular limb motions (Carello, Kinsella-Shaw, Amazeen, \& Turvey, 2006; Carello \& Turvey, 2004), and the wielding of tools (Cabe, 2011). Sighted, sighted-blindfolded, and early-blind observers have shown similar spatial abilities on the three-mountain elevation task (Heller \& Kennedy, 1990; Kennedy, 1993). However, experiments on the three-mountain task have only asked for one plan - the one from the observer's side of the array of objects. From opposite the observer, the plan in Fig. 1b would be redrawn with the cone as the lowest form, and the circle would be on the right side of the plan. For a vantage point on the left side of Fig. 1b, the plan would have the square in the middle and high. From the right, the square would be middle and low. Each vantage point has a unique plan as well as a unique elevation. Also, it is worth noting that the task of reporting a vantage point other than one's own might be undertaken using "mental rotation" (Sathian \& Prather, 2006; Shepard \& Metzler, 1971), and the three-mountain task is perspective taking because it requires people to imagine a different perspective from their own.

Broadly speaking, assessed for accuracy, blind, sighted, and blindfolded-sighted adults are often reported to have similar spatial abilities, but different response speeds have been noted. All three groups successfully report the directions of objects in many kinds of elevation tasks (Haber, Haber, Penningroth, Novak, \& Radgowski, 1993). For example, they can turn to face and point to targets after walking toward or away from them (Horn \& Loomis, 2004; Kennedy, 1993; Loomis \& Philbeck, 2008). After learning four-point routes from tactile or visually inspected maps, blind and sighted adults were asked to imagine perspectives aligned or misaligned with maps they studied (Giudice, Betty, \& Loomis, 2011). Blind and sighted participants had similar patterns of errors and latencies. Giudice, Klatzky, Bennett, and Loomis (2013) found that blindfolded-sighted observers using canes or touching targets with their bare hands, who learned the locations of 24 different target locations in a 2-m radius from a fixed origin, fairly accurately located them again from novel vantage points to which they were walked. Evidently, like direct contact, "extended touch" with probes allows 3-D spatial appreciation. On the three-mountain task, Heller and Kennedy (1990) tested a sighted group, a sighted-blindfolded group, an adventitiously blind group, and a congenitally blind group. The participants identified or drew raised-line pictures from five perspectives (four elevations and the plan task from the observer's vantage point). The congenitally blind participants performed nearly as accurately as the sighted and adventitiously blind participants, although they took more time to finish. In a three-mountain test of congenitally and adventitiously blind adults, similar accuracy-response-time trade-offs have been reported by Ruggiero, Ruotolo, and Iachini (2012), and, again, the accuracy of the congenitally blind adults was comparable to that of the blindfolded-sighted. Ruggiero et al. concluded that overall performance was quite good and, likely, any differences were quantitative rather than qualitative. Also, use of the observer's vantage point is evident in drawings by sighted and blind adults; for example, converging lines show sides of objects receding from the vantage point (Kennedy \& Juricevic, 2006a, 2006b). The blind participants in these studies used raised-line pictures (Eriksson, 1998; Hatwell \& Martinez-Sarrochi, 2003; Picard \& Lebaz, 2012). Also, blind and sighted adults recognize foreshortened pictures of objects fitting a single vantage point to the side of an array of objects (Heller et al., 2006; Heller et al., 2009). In making drawings of houses, the order of success was "from above," then "from the side," and then "three-quarter views" (Heller, Kennedy, \& Joyner, 1995) - that is, plan, then elevation, and then three-quarter views. Three groups of participants (blindfolded sighted, late-blind, and congenitally blind) were shown a board tilted at different angles (Heller, Calcaterra, Tyler, \& Burson, 1996). The groups correctly selected foreshortened drawings at similar rates. Both blind groups performed better than the blindfoldedsighted on drawings of vertical panels - that is, elevations.

To date, experiments on the three-mountain task have used only the plan task from the observer's side. However, Eardley and Van Velzen (2011) reported that blind adults use external spatial frames of reference (allocentric reference) in attending
Fig. 1 a Elevation task: the order of three objects from the observer's vantage point. b Plan task: the arrangement of three objects from the observer's vantage point and above the scene

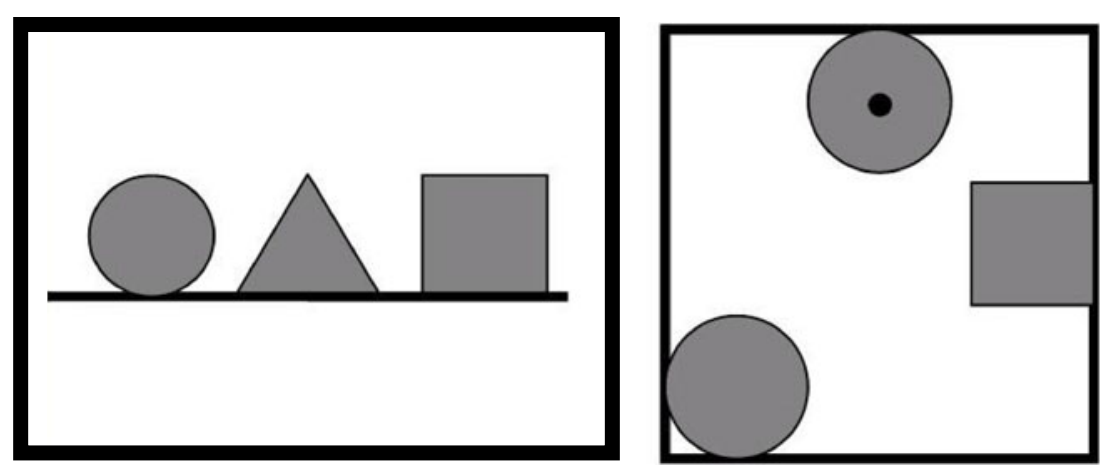

(a) (b) 
Table 1 Four groups of participants with different testing conditions

\begin{tabular}{lllll}
\hline Groups & $\begin{array}{l}\text { Always } \\
\text { sighted }\end{array}$ & $\begin{array}{l}\text { Sighted first, } \\
\text { blindfolded later }\end{array}$ & $\begin{array}{l}\text { Always } \\
\text { blindfolded }\end{array}$ & Early-blind \\
\hline$N$ & 24 & 24 & 19 & 6 \\
Mean age & 19.5 & 18.7 & 18.7 & 51.8 \\
Age $S D$ & 2.4 & 2.0 & 2.6 & 10.2 \\
\hline
\end{tabular}

to target locations in plan tasks. Blind adults show good understanding of different kinds of 2-D arrays representing spaces (Millar, 1994; Picard \& Lebaz, 2012; Picard, Lebaz, Jouffrais, \& Monnier, 2010) —in particular, small-scale plans showing large-scale 2-D spaces (Hatwell \& MartinezSarrochi, 2003; Tinti, Adenzato, Tamietto, \& Cornoldi, 2006).

Evidently, sighted and blind adults undertake some perspective-taking tasks successfully. However, Picard and Lebaz (2012, p. 430) suggested that "visually realistic" projections of solid, bulky 3-D objects with $z$-dimension extents in 2-D tactile drawings are more difficult for the blind to identify than are similar drawings showing flat objects. Eriksson (1998) noted that projection of 3-D scenes into 2-D pictures has often been considered unrealistic for blind people. Heller and Kennedy (1990) found performance times of their congenitally blind group to be especially long, and response time might measure difficulty. Elevations may entail projections of objects at different $z$-dimension distances. If so, blind people might find some perspective-taking tasks difficult, including tasks that the sighted deem relatively easy.

The left-right azimuth order of the objects from a particular vantage point is shown in an elevation. A plan shows the leftright order and the relative distance (near-middle-far) of the objects. Both vision and touch can discover these spatial orders. An elevation can be considered as a projection that preserves left-right information. A plan preserves distance information in addition. It can also be considered to be a

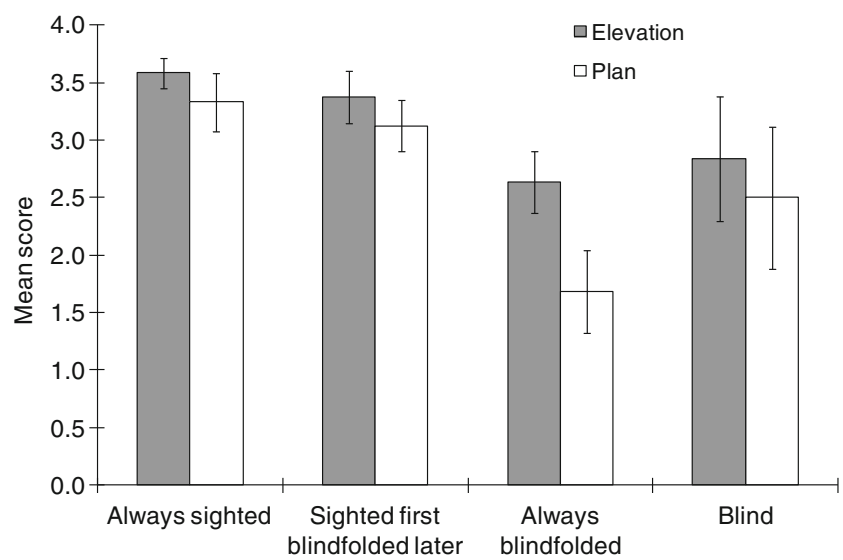

Fig. 2 Performance of each group on elevation and plan tasks. Error bars are standard errors projection, one that is to a vantage point above target objects. If so, both are projections - not different in that respect.

Perhaps the key factor is the information preserved in elevations and plans. An elevation requires participants only to arrange objects in one order - namely, left-right - and a plan requires both left-right and near-far information to be shown. Since it requires more information to be presented, a plan task could be more difficult than an elevation task. It might result in lower accuracy scores. Furthermore, the observer may judge that more processing is required to present the plan response and may report that it is more difficult. Further still, observers who are equally accurate on plan and elevation tasks may recognize that plans require presenting more information and may judge them more difficult. These hypotheses apply equally to sighted, blindfolded-sighted, and blind participants.

In sum, we ask how early-blind, sighted and sighted adults respond to Piaget three-mountain tasks, given four plan tasks and four elevation tasks. We report on accuracy and on judgments of difficulty.

\section{Method}

\section{Participants}

Seventy-three participants were tested in four conditions (Table 1). Sighted participants were volunteers from an introductory psychology class at the University of Toronto, Scarborough, given course credit for participation. All had normal eyesight, with or without correction. An alwayssighted group had 24 participants (12 male); a sightedfirst-and-blindfolded-later group had 24 (9 male); an always-blindfolded group had 19 (7 male). The early-blind group had 6 participants (3 male), recruited from Canadian organizations for the blind in Toronto and Ottawa. All were totally blind prior to 3 years old. The groups were not age

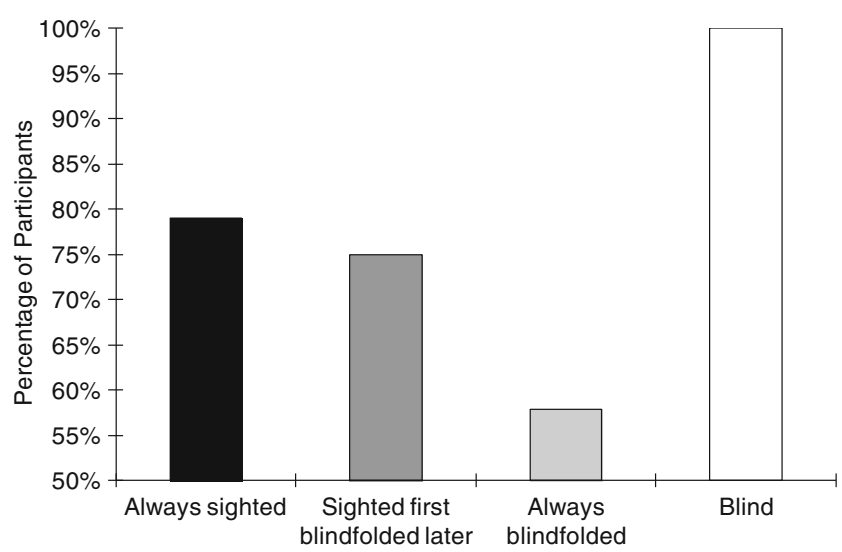

Fig. 3 Percentage of "plan is more difficult" judgments per group 
matched. (Finding early-blind volunteers equal in age to firstyear students is difficult.)

\section{Apparatus}

Three wooden geometric 3-D objects, an $8.5-\mathrm{cm}$ cube, a sphere $8.5 \mathrm{~cm}$ in diameter, and a cone $(8.5 \mathrm{~cm}$ wide base and height), were placed on a $30 \times 30 \mathrm{~cm}$ flat board on a table in front of participants, as shown in Fig. 1. To remove effects caused by differences in experience or skill in making drawings, participants were given flat shapes that could be arranged on response boards to represent the array of 3-D objects. The flat shapes were 2-D geometric shapes made of 0.5 -cm-thick cards, four $5.5-\mathrm{cm}$ squares, four circles $5.5 \mathrm{~cm}$ in diameter, four equilateral triangles $5.5 \mathrm{~cm}$ per side, and four circles $5.5 \mathrm{~cm}$ in diameter with a dot in the center. In the elevation task, the response board was a rectangular card $(30 \times 25.5 \mathrm{~cm})$ that had four parts $7.5 \times 25.5 \mathrm{~cm}$ each. On the four parts, participants could arrange the flat shapes to show the 3-D objects from four vantage points. In the plan task, participants were asked to place the flat forms on four rectangular boards $(21.5 \times 20 \mathrm{~cm})$ to represent the four possible plans. The boards and flat shapes were white. All were made of material similar to Bristol board.

In the test, participants picked 2-D geometric shapes and constructed plans and elevations. Participants in one sighted group saw the stimuli first and were blindfolded later, and always-blindfolded participants used the blindfold after seeing the flat objects and response boards but before being shown the array of 3-D objects to be depicted.

\section{Procedure}

Three geometric objects were put on a rectangular table as in Fig. 1. The participant was seated on one side, eye position at approximately $45^{\circ}$ to the middle of the horizontal flat boardthat is, not directly above or level with the tabletop. First, participants saw or were guided to touch the three 3-D objects and the 2-D shapes. They were instructed that they should place the 2-D shapes on response boards to show the objects. Half of the participants were given a board for the elevation task first; the rest were given boards for the plan task first. In each task, the different vantage points at issue were given in random orders, with the restriction that the participants began with their own vantage point in both the plan and elevation tasks.

The vantage point to be used was pointed out manually to sighted participants in the condition in which they were being shown the displays with no blindfold. Dolls $18 \mathrm{~cm}$ tall were placed at the relevant vantage point for blind and blindfolded participants, who reached out to touch the dolls to ascertain their relative viewpoint. They were free to reach out to the objects once the doll had been positioned. Participants were asked to place the response shapes on the boards to show the array of objects from the doll's vantage point. To show the plan view required, the doll's head was placed above the center of the 3-D object array with its feet directed toward the relevant vantage point. To show an elevation vantage point, the doll was placed to one side of the array of objects, feet on the table. There was no time limitation during testing, but the duration of the experiment was generally about 20 min. No feedback was given until debriefing after the experiment was completed.

Responses were scored correct if the elevation order was correct left-to-right. Plan views were correct if left-right and near-far order was correct. A score of "1" was awarded for the correct answer, and " 0 " for incorrect. Hence, on each task, the maximum was 4.

After completing the plan and elevation tasks, participants were asked: Which task is more difficult for you? With the doll above the array of objects or to the side of the objects?

\section{Results}

Figure 2 shows the mean plan and elevation scores, and Fig. 3 shows the percentage who judged the plan task as more difficult than the elevation task.

On a mixed-measures ANOVA, with experimental group (always-sighted, always-blindfolded, sighted-firstblindfolded-later, early-blind) as the between factor and task (plan or elevation) as the within factor, elevation scores were higher $(M=3.20, S D=0.12$; plan $M=2.77, S D=0.16)$, $F(1,69)=7.75, p=.007$. Overall scores were different across the groups, $F(3,69)=6.71, p<.001$, with the alwaysblindfolded scoring lower overall $(M=2.16, S D=1.19)$ than either the always-sighted $(M=3.46, S D=0.87 ; p=.001)$ or the sighted-first-blindfolded-later $(M=3.25, S D=0.98$; $p=.005)$. The early-blind group $(M=2.67, S D=0.57)$ was intermediate and not reliably different from any sighted group (all $p \mathrm{~s}>.05$ ). The interaction between task and experimental group was not significant, $p>.05$.

Differences between the tasks in judgments of difficulty were assessed using the binomial test. Fifty-four of the 73 participants judged the plan task more difficult $(z=3.98, p<.001)$. The effect was present in three of the groups, the exception being the always-blindfolded adults. Nineteen out of 24 sighted $(z=2.65, p=.007), 18$ out of 24 sighted-first-and-blindfolded later $(z=2.25, p=.02)$, and all 6 early-blind $(p=.03)$ participants judged the plan task more difficult. However, only 11 out of 19 always-blindfolded adults thought the plan task was more difficult $(z=0.46, p=.65)$. That is, the three groups that scored highest and obtained fairly similar scores on the two tasks judged the plan task relatively difficult. Furthermore, in these three groups, of the participants who scored the same on the two tasks, 11 out of 12 always-sighted 
( $z=2.6, p=.003$, one-tailed binomial), 10 out of 13 sighted-firstand-blindfolded later $(z=1.7, p=.05$, one-tailed binomial), and 4 out of 4 early-blind participants $(p=.06$, one-tailed binomial) judged the plan task more difficult.

\section{Discussion}

Of interest, the groups' scores ranged from close to ceiling (4) to not far from 1, which would be chance if there were only four options. However, the scores are above chance if all 6 elevation combinations and all 36 plan combinations are relevant. In general, plan scores are lower than elevation scores. The lowest mean score is $1.7(S D=1.6$; blindfolded group, plan task), and the near-ceiling score is $3.6(S D=0.7$; always sighted, elevation task). Usefully, the Piaget task can strain adults, although in some conditions, it is straightforward, and thereby it provides enough range to be sensitive. The lowest-score condition involves a plan task, and the highest-score condition an elevation, but it may be the amount of spatial-order information determines difficulty, rather than plan versus elevation per se.

The early-blind group's scores are not reliably different from those of the other groups, but it is noteworthy that the early-blind group's scores (elevation, $M=2.8$; plan, $M=2.5$ ) on both tasks were numerically above the scores of the alwaysblindfolded (elevation, $M=2.6$; plan, $M=1.7$ ). (In a pilot study, a group of 2 early-blind and 6 totally-blind volunteers who went blind after age 3 produced results similar to those of the early-blind group reported here.) Furthermore, the spatial skills of the early-blind are comparable to those of the sighted, since their mean score is not significantly different from that of any other group. Also, for each group, including the earlyblind, scores on elevation tasks were numerically higher than those on plan tasks, supporting the hypothesis that similar challenges were faced by all the groups.

From a particular vantage point, the highest possible mean score is 1 . The highest means were for the observer's vantage point (.92), next the opposite vantage point (.7), and lastly the vantage point to the left (.64) and right (.64), equally. As compared with the observer's vantage point, the opposite vantage point elevation of Fig. 1 involves the central object remaining constant and a mere switching of two objects. The left vantage point involves more changes to the elevation-for example, not only an object on the left moving to the right, but also the middle becoming the left object. The right vantage point elevation leaves the rightmost object (a circle) constant but switches the others (square and triangle), which may not be as obvious as leaving the center constant. Considering the plan and elevation scores separately, the lowest mean was for the early-blindfolded group in the plan condition (.26), and no other group scored less than .3. Of interest, the early-blind group scored a perfect 1.0 on the elevation task from the observer's vantage point (equal with the always-sighted group) and obtained the highest score on the elevation task from the vantage point opposite the observer (.83), with the alwayssighted and blindfolded-later group close behind (.79 each). Evidently, the early-blind group fits in ranges defined by the groups using vision and has the same pattern of results as the groups using vision.

The plan task was judged harder than the elevation task by all groups, and the differences were significant for three groups, including the early-blind. Even participants scoring equally on the tasks judged the plan more difficult. Is there an apparent mismatch between these equal-accuracy scores and different subjectively perceived difficulty? Perhaps there is not. More information is presented in the plan task responses, and this could influence judgments of difficulty. By analogy, multiplying 35 by 7 requires juggling more information than does multiplying 31 by 7 , even if observers are equally correct on both multiplications. Always-blindfolded participants scored lowest on the tasks and may have found both tasks sufficiently difficult that they could not distinguish their degrees of difficulty. In sum, both accuracy and subjective judgments of difficulty from three higher-accuracy groups indicate that the plan task was relatively challenging.

Projection to the side that treats distance information as irrelevant did not make elevations especially hard even for the early-blind. The early-blind participants make the same judgments about difficulty as most of the sighted. Plans may be relatively difficult because they require participants to coordinate two dimensions $-x$ and $z$ dimensions. In elevations, only one dimension is controlled - the $x$ dimension. That is, the scoring system makes the two views nonequivalent in terms of difficulty. Elevation responses have to be correct only from left to right. Plans have to be correct left to right and near to far.

Observers may always be aware of the dimensions of the display but find it easier to output one of its dimensions. If so, the bottleneck that controls difficulty lies in the response mechanism and task decomposition, and not in the cognition of the distal stimulus array (Hegarty, 2010). In other words, observers may know the left-right and distance coordinates of the cube, sphere, and cone, but in responding they have to ask only "do I put the square on the right?" rather than "do I put it on the right, and do I put it near, middle, or far." Downloading one dimension of one object, such as the square, and then shifting attention to another object, such as the cone, may be easier than asking for two dimensions for an object before considering the next object. The elevation task has two degrees of freedom, and the plan task has four. That is, once the square and triangle are assigned left-right positions in an elevation, only one slot is available for the circle. In a plan, the square and triangle have to be assigned left-middle-right and low-middle-high positions before only one position is open for the circle.

Some cautions are in order. Further research might usefully address matters of scale or distance, in addition to order of 
targets, and use other vantage points - for example, at the corners of the $30 \times 30 \mathrm{~cm}$ board. Given that some of the scores are near ceiling, if a more fine-grained, or spatial rather than ordinal, scoring system were used that took into account not only the order, but also the relative locations, spatial relations, and distances among the targets, subtle differences or trends might be revealed that are not visible with the present scoring method.

Individual-difference and developmental questions (Wolbers \& Hegarty, 2010) deserve attention. Landau, Gleitman, and Spelke (1981), in a pioneering study, found that a congenitally blind $2 \frac{1}{2}$-year-old could determine the path between two objects after walking to each from a third object. This suggests awareness of a plan of the object layout and, hence, the directions from each object to any other, from infancy. If so, novices at the three-mountain task, and children in particular, might find plan views relatively easy from their vantage point. Furthermore, treating one dimension of a stimulus, such as near-far, as irrelevant may not be immediately acceptable to novices and children. One reason for thinking this is that cubes are often drawn by novices and 6- to 8-year-old children as if folded out, showing four or five sides, as if representation requires showing many aspects of a stimulus (Kennedy \& Hammad, 2012).

Studies on early-blind individuals often involve concerns about statistical power. Profound visual impairment from early infancy is rare. As a result, we acknowledge that low numbers, high $S D$ s, and a lack of difference between the earlyblind and the blindfolded groups raise issues of power that testing large groups would solve. That the early-blind group here was intermediate between sighted groups is helpful. If the early-blind had been lower or higher than all of the other groups, nonsignificant differences would have made power issues a larger concern.

Another caution has to do with foreshortening. The form of projection that may be relevant to the present tasks does not involve diminution with distance - that is, linear perspective. Rather, it could be considered to be parallel projection, in which the sizes of the 2-D forms used to depict the 3-D objects are fixed despite near-far variation. Further research could determine whether elevations changing in 2-D size to depict distance are especially challenging. They may provide more difficulty than do plans, which use squares, triangles, and circles with fixed sizes and represent depth by locations in a 2-D quadrilateral. In a plan, a distance in 2-D represents the $z$-distance of the object from the observer. One distance representing another distance may be easier than size representing distance. Diderot argued that the blind can enlarge or shrink shapes mentally (Kennedy, 1993; Kennedy, Gabias, \& Heller, 1992). However, Diderot was concerned with real objects, big and small, of similar shapes (the world and a ball). He was not considering a $z$-distance in 3-D represented by size change on a 2-D picture surface.
Besides 2-D size, foreshortening changes angles. Most sighted adults have difficulty determining how to draw tilted squares in perspective by adjusting angles at vertices (Kennedy, 1993), and the early-blind likely have similar issues, even if they are very familiar with cubes at many orientations (Eriksson, 1998; Heller et al., 2006; Heller et al., 2009; Kennedy, 1993; Picard \& Lebaz, 2012). This is a topic that deserves considerable investigation.

In sum, early-blind and sighted groups were tested on plan and elevation tasks using a Piaget three-mountain display. Both found the elevation tasks easier, which could be due to response factors having to do with task decomposition and rule following (Hegarty, 2010), since the elevation task requires responses controlling left-right order and the plan task requires responses controlling near-far as well as left-right.

Acknowledgments National Science Council of Taiwan (NSC972917-I-011-102), National Gallery of Canada, Alliance for Equality of Blind Canadians, Vision Impaired Ministry (Taiwan), Dr. Chih-Ming Shih Kung-Ching Chen, Sherief Hammad, Justin Deonarine, Jack Loomis, Morton Heller, and two AP\&P reviewers.

\section{References}

Cabe, P. A. (2011). Haptic distal spatial perception mediated by strings. Journal of Experimental Psychology. Human Perception and Performance, 37, 1492-1511.

Carello, C., Kinsella-Shaw, J., Amazeen, E., \& Turvey, M. T. (2006). Peripheral neuropathy and object length perception by effortful (dynamic) touch: A case study. Neuroscience Letters, 405, 159-163.

Carello, C., \& Turvey, M. T. (2004). Physics and psychology of the muscle sense. Current Directions in Psychological Science, 13, 25-28.

Eardley, A. F., \& Van Velzen, J. (2011). Event-related potential evidence for the use of external coordinates in the preparation of tactile attention by the early-blind. European Journal of Neuroscience, 33, 1897-1907.

Eriksson, Y. (1998). Tactile pictures: Pictorial representations for the blind. Gothenburg, Sweden: Gothenburg University Press.

Giudice, N. A., Betty, M. R., \& Loomis, J. M. (2011). Functional equivalence of spatial images from touch and vision: Evidence from spatial updating in blind and sighted individuals. Journal of Experimental Psychology: Learning, Memory, and Cognition, 37, 621-634.

Giudice, N. A., Klatzky, R. L., Bennett, C. R., \& Loomis, J. M. (2013). Perception of 3-D location based on vision, touch, and extended touch. Experimental Brain Research, 224, 141-153.

Haber, L., Haber, R. N., Penningroth, S., Novak, K., Radgowski, H. (1993). Comparison of nine methods of indicating the direction to objects: data from blind adults. Perception, 22, 35-47

Hatwell, Y., \& Martinez-Sarrochi, F. (2003). The tactile reading of maps and drawings and the access of blind people to works of art. In Y. Hatwell, A. Streri, \& E. Gentaz (Eds.), Touching for knowing (pp. 255-273). Amsterdam: John Benjamins.

Hegarty, M. (2010). Components of spatial intelligence. In B. H. Ross (Ed.), The psychology of learning and motivation (Vol. 52, pp. 265-297). San Diego: Academic Press.

Heller, M. A., Calcaterra, L. A., Tyler, L. A., \& Burson, L. L. (1996). Production and interpretation of perspective drawings by blind and sighted people. Perception, 25, 321-334. 
Heller, M. A., \& Kennedy, J. M. (1990). Perspective taking, pictures, and the blind. Perception \& Psychophysics, 48, 59-466.

Heller, M. A., Kennedy, J. M., Clark, A., McCarthy, M., Borgert, A., Wemple, L., ... Riddle, T. (2006). Viewpoint and orientation influence picture recognition in the blind. Perception, 35, 13971420.

Heller, M. A., Kennedy, J. M., \& Joyner, T. D. (1995). Production and interpretation of pictures of house by blind people. Perception, 24, 1049-1058.

Heller, M. A., Riddle, T., Fulkerson, E., Wemple, L., Walk, A. M., Guthrie, S., ... Klaus, P. (2009). The influence of viewpoint and object detail in blind people when matching pictures to complex objects. Perception, 38(8), 1234-1250.

Horn, D. L., \& Loomis, J. M. (2004). Spatial updating of targets in front and behind. Paidéia, 14, 75-81.

Kennedy, J. M. (1993). Drawing and the blind. New Haven, CT: Yale University Press.

Kennedy, J. M., Gabias, P., \& Heller, M. A. (1992). Space, haptics and the blind. Geoforum, 23(2), 175-189.

Kennedy, J. M. \& Juricevic, I. (2006a) Blind man draws using diminution in three dimensions. Psychonomic Bulletin and Review, 13, 506-509.

Kennedy, J. M. \& Juricevic, I. (2006b) Foreshortening, convergence and drawings from a blind adult. Perception, 35, 847-851.

Kennedy, J. M., \& Hammad, S. (2012). Cognition and representation: Picture perception. In A. L. Blum, J. M. Krois, \& H.-J. Rheinberger (Eds.), Verkoerperungen ("Embodiment”) (pp. 71-83). Berlin: Akademie Verlag.

Klatzky, R., \& Lederman, S. (2003). The haptic identification of everyday objects. In Y. Hatwell, A. Streri, \& E. Gentaz (Eds.), Touching for knowing (pp. 105-123). Amsterdam: Benjamins.

Landau, B., Gleitman, H., \& Spelke, E. (1981). Spatial knowledge and geometric representation in a child early-blind from birth. Science, 213, 1275-1278.

Loomis, J. M., \& Philbeck, J. W. (2008). Measuring perception with spatial updating and action. In R. L. Klatzky, M. Behrmann, \& B. MacWhinney (Eds.), Embodiment, ego-space and action (pp. 1-43). Mahwah, NJ: Erlbaum.
Millar, S. (1994). Understanding and representing space: Theory and evidence from studies with blind and sighted children. Oxford: Oxford University Press, Clarendon Press.

Piaget, J. (1954). The construction of reality in the child. New York: Basic Books.

Piaget, J., \& Inhelder, B. (1956). The child's conception of space. The coordination of perspectives, pp. 209-246. (F. J. Langdon \& J. L. Lanzer, Trans.). New York: Norton. (Original work published in 1948).

Picard, D., \& Lebaz, S. (2012). Identifying raised-line drawings by touch: A hard but not impossible task. Journal of Visual Impairment \& Blindness, 106, 427-431.

Picard, D., Lebaz, S., Jouffrais, C., \& Monnier, C. (2010). Haptic recognition of two-dimensional raised-line patterns by earlyblind, late-blind, and blindfolded sighted adults. Perception, 39, 224-235.

Ruggiero, G., Ruotolo, F., \& Iachini, T. (2012). Egocentric/allocentric and coordinate/categorical haptic encoding in early-blind people. Cognitive Processing, 13(Suppl. 1), 313-317.

Sathian, K., \& Prather, S. C. (2006). Cerebral cortical processing of tactile form: Evidence from functional neuroimaging. In M. A. Heller \& S. Ballesteros (Eds.), Touch and blindness: Psychology and neuroscience (pp. 157-170). Lawrence Erlbaum Assosciates: Mahwah, N. J.

Shepard, R. N., \& Metzler, J. (1971). Mental rotation of threedimensional objects. Science, 171, 701-703.

Soska, K. C., \& Johnson, S. P. (2008). Development of three-dimensional object completion in infancy. Child Development, 79(5), 1230 1236.

Tinti, C., Adenzato, M., Tamietto, M., \& Cornoldi, C. (2006). Visual experience is not necessary for efficient survey spatial cognition: Evidence from early-blindness. The Quarterly Journal of Experimental Psychology, 59, 1306-1328.

Vandoorn, G., Hohwy, J., \& Symmons, M. A. (2012). Capture of kinesthesis by a competing cutaneous input. Attention, Perception, \& Psychophysics, 74, 1539-1551.

Wolbers, T., \& Hegarty, M. (2010). What determines our navigational abilities? Trends in Cognitive Sciences, 14, 138-146. 